\title{
Regularized Tomographic Density Imaging Using Multiple Frequency Information
}

\author{
Roberto J. Lavarello ${ }^{*}{ }^{\dagger}$, Stephen D. Bond ${ }^{\ddagger}$, and Michael L. Oelze* \\ * Bioacoustics Research Laboratory, Department of Electrical and Computer Engineering \\ University of Illinois at Urbana-Champaign, Urbana, IL 61801 \\ Email: lavarell@illinois.edu \\ † Laboratorio de Imágenes Médicas, Departamento de Ingeniería \\ Pontificia Universidad Católica del Perú, San Miguel, Lima 32, Perú \\ $\ddagger$ Applied Mathematics Division, Sandia National Laboratories, Albuquerque, NM 87185
}

\begin{abstract}
Inverse scattering methods for density imaging have limitations in terms of required signal-to-noise ratio and bandwidth that keep them from being experimentally implemented. The multiple frequency distorted Born iterative method (MFDBIM), has been previously proposed to overcome some of these limitations. The objective of this work is to study the convergence of MF-DBIM through both simulations and experiments. Simulations were conducted by reconstructing circular cylinders of radii 1,2 , and 4 wavelengths, and ratios of density $\Delta \rho$ to sound speed $\Delta c$ contrasts between -3 and 2. Experiments were performed using a balloon phantom filled with saline and frequencies between 1.5 and $3 \mathrm{MHz}$. Two methods for stabilizing MF-DBIM were studied: total variation regularization (TVR), and weighted TVR giving emphasis to the variation of the pixels around the edges of the imaging target. In simulations, the convergence of MF-DBIM was found to be dependent on the imaging target. For cylinders with $\Delta \rho / \Delta c<0$ reconstruction errors were typically below $30 \%$. The errors were significantly higher (i.e., up to $70 \%$ minimum reconstruction error) for cylinders with $\Delta \rho / \Delta c>0$. The degraded performance of MFDBIM was related to the limited spatial bandwidth of the inverse scattering problem. In experiments, calibration errors did not allow reconstruction of useful density tomograms when using MF-DBIM. Density tomograms with $56 \%$ reconstruction errors were obtained with MF-DBIM and TVR, but only for a very narrow range of regularization parameters. In contrast, reconstruction errors between $55 \%$ and $60 \%$ were obtained with MF-DBIM and weighted TVR for regularization parameter values spanning more than an order of magnitude. Therefore, preliminary experimental results presented here suggest auxiliary techniques such as weighted TVR may help extending the convergence of tomographic density imaging algorithms.
\end{abstract}

\section{Motivation}

Acoustic tomography is a quantitative imaging technique that aims to reconstruct material properties based on scattered pressure measurements. Typically, density changes are neglected in order to obtain estimates of speed of sound and attenuation [1]. However, actual values of density and compressibility are not known for many disease states and therefore determining density distributions may provide additional information or contrast in imaging for cancer detection.

Inverse scattering methods for density imaging can be roughly classified in two categories. The first approach consists of inverting the wave equation by solving for a single functional that depends on both speed of sound and density variations, and using data at two frequencies to isolate density information [2]. The second approach consists of solving the wave equation for two functionals simultaneously: one that depends only on compressibility and one that depends only on density variations [3]. It has been previously reported that both approaches have limitations that keep them from practical experimental implementations [4]. In particular, the first approach (as exemplified by the dual frequency distorted Born iterative method, DF-DBIM) was found to be very sensitive to the termination tolerance of the single frequency reconstructions, and the second approach (as exemplified by the T-matrix approach) was found to require a very large bandwidths. An improved approach, the multiple frequency DBIM (MF-DBIM) algorithm [5], has been previously proposed to overcome some of these limitations. The objective of this work is to study the convergence of MF-DBIM through both simulations and experiments.

\section{METHODS}

\section{A. Variable density and the distorted Born iterative method}

The inhomogeneous wave equation can be written as [4]

$$
p(\vec{r})=e_{s}(\vec{r})+\int_{\Omega} d \vec{r}^{\prime} \mathcal{O}\left(\vec{r}^{\prime}, \omega\right) p\left(\vec{r}^{\prime}\right) G_{0}\left(\vec{r}, \vec{r}^{\prime}\right),
$$

where $p(\vec{r})$ is the acoustical pressure, $e_{s}(\vec{r})$ is the incident field caused by a source located at $\vec{r}_{s}, s=0,1, \ldots, N_{s}$, and $G_{0}\left(\vec{r}, \vec{r}^{\prime}\right)$ is the Green's function in a homogeneous background with wave number $k_{0}$. Assuming the speed of sound $c(\vec{r})$ is weakly dispersive and neglecting attenuation for simplicity, the object function $\mathcal{O}(\vec{r}, \omega)$ is given by

$$
\mathcal{O}(\vec{r}, \omega)=\left[\frac{\omega^{2}}{c^{2}(\vec{r})}-\frac{\omega^{2}}{c_{0}^{2}}\right]-\rho^{1 / 2}(\vec{r}) \nabla^{2} \rho^{-1 / 2}(\vec{r}) .
$$

where $\rho(\vec{r})$ is the density distribution, $\omega$ is the angular frequency of the incident harmonic wave, and $c_{0}$ is the sound speed in the background. Equation (1) was discretized using sinc basis functions and delta testing functions. The DBIM [6] is an algorithm that estimates $\mathcal{O}(\vec{r}, \omega)$ using (1) and measurements of the scattered field $p^{s c}(\vec{r})=p(\vec{r})-e_{s}(\vec{r}), \vec{r} \notin \Omega$. A trial $\overline{\mathcal{O}}_{(0)}$ is chosen for which the corresponding scattered field is calculated. Next, the object function is updated as 
$\overline{\mathcal{O}}_{(n+1)}=\overline{\mathcal{O}}_{(n)}+\Delta \overline{\mathcal{O}}_{(n)}$, where $\Delta \overline{\mathcal{O}}_{(n)}$ is given by the regularized optimization problem

$$
\Delta \mathcal{O}_{(n)}=\underset{\Delta \mathcal{O}}{\arg \min }\left\|\Delta \bar{p}^{s c}-\bar{F}_{(n)} \cdot \Delta \mathcal{O}\right\|_{2}^{2}+\gamma\|\Delta \mathcal{O}\|_{2}^{2},
$$

where $\Delta \bar{p}^{s c}$ contains the difference between the predicted and measured scattered fields, $\gamma$ is the regularization parameter, and $\bar{F}_{(n)}$ is the so-called Frechet derivative matrix [1]. The iterative process is repeated until the relative residual error (RRE), given by $\mathrm{RRE}=\left\|\Delta \bar{p}^{s c}\right\|_{2} /\left\|\bar{p}^{s c}\right\|_{2}$, falls within a desired termination tolerance $t \%$.

\section{B. The multiple frequency DBIM (MF-DBIM) algorithm}

If a set of reconstructions $\mathcal{O}_{i}(\vec{r})=\mathcal{O}\left(\vec{r}, \omega_{i}\right)$ at frequencies $\omega_{i}, i=1,2, \ldots, N_{f}$ are available, the least mean squares estimator

$$
\mathcal{F}_{\rho}(\vec{r})=\frac{\left(\sum_{i=1}^{N_{f}} \omega_{i}^{2}\right)\left(\sum_{i=1}^{N_{f}} \omega_{i}^{2} \mathcal{O}_{i}(\vec{r})\right)-\left(\sum_{i=1}^{N_{f}} \omega_{i}^{4}\right)\left(\sum_{i=1}^{N_{f}} \mathcal{O}_{i}(\vec{r})\right)}{N_{f} \sum_{i=1}^{N_{f}} \omega_{i}^{4}-\left(\sum_{i=1}^{N_{f}} \omega_{i}^{2}\right)^{2}}
$$

allows for the isolation of density contributions $\mathcal{F}_{\rho}=$ $\rho^{1 / 2}(\vec{r}) \nabla^{2} \rho^{-1 / 2}(\vec{r})$ to the object function. After estimating $\mathcal{F}_{\rho}$, density images can be constructed by solving

$$
\left(\mathcal{L}-\mathcal{D}\left(\bar{F}_{\rho}\right)\right) \cdot \bar{u}=\bar{G} \cdot \bar{u}=\bar{F}_{\rho}
$$

where $\bar{u}$ is a vector with the values of $u(\vec{r})=\left(\rho_{r}^{-1 / 2}(\vec{r})-1\right)$, $\bar{F}_{\rho}$ is a vector with the values of $\mathcal{F}_{\rho}(\vec{r}), \mathcal{L}$ is a matrix approximation of the $\nabla^{2}$ operator, and $\mathcal{D}$ is an operator that transforms a vector into a diagonal matrix.

Frequency hopping [7], i.e., the use of lower frequency reconstructions as initial guesses for higher frequency reconstructions, was used to improve on the cancellation of the speed of sound term in (2). The DBIM initial guess when processing data at frequency $\omega_{i}$ was chosen as

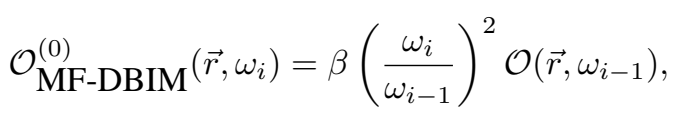

where where $\mathcal{O}\left(\vec{r}, \omega_{i-1}\right)$ is the final reconstruction obtained at the nearest lower frequency $\omega_{i-1}$ and $\beta=(1-1.5 t \%)$.

The multiple frequency DBIM (MF-DBIM) [5] consists of using (4) with profiles obtained at frequencies $f \in$ $\left[f_{\min }, f_{\max }\right]$ in steps of $\Delta f=\frac{f_{\max }-f_{\min }}{N_{f}-1}$, and (6) as DBIM initial guess. A conventional 2D median filter of size 3 by 3 pixels was used to smooth $\mathcal{O}_{\mathrm{MF}-\mathrm{DBIM}}^{(0)}{ }^{\left(\vec{r}, \omega_{i}\right) \text {. Frequency }}$ hopping improves on the correlation among all profiles except the one obtained at frequency $f_{\min }$, which is obtained using an all-zero initial guess and therefore requires special treatment. In this work, the data at $f_{\min }$ was processed twice. The first time a profile $\mathcal{O}^{\prime}\left(\vec{r}, \omega_{\min }\right)$ was obtained by using an all-zero initial guess. The second time, the final profile $\mathcal{O}\left(\vec{r}, \omega_{\text {min }}\right)$ was obtained by using $\beta \mathcal{O}^{\prime}\left(\vec{r}, \omega_{\text {min }}\right)$ as initial guess.

\section{Regularized MF-DBIM}

Regularization can be used to stabilize the solution of (5). The generalized Tikhonov regularization consists of solving the optimization problem [8]

$$
\hat{u}=\underset{\bar{u}}{\arg \min }\left\|\bar{F}_{\rho}-\bar{G} \cdot \bar{u}\right\|_{2}^{2}+\gamma_{R} \sum_{i=1}^{N}\left(\left|(\bar{L} \cdot \bar{u})_{i}\right|^{2}+\beta\right)^{k / 2},
$$

where $\gamma_{R}$ is a regularization parameter and $\beta$ is a small positive constant $\left(\beta=10^{-10}\right)$ introduced to avoid gradient singularities. The solution $\hat{u}$ to Eq. (7) is given by

$$
\hat{u}=\left[\left(\bar{G}^{H} \cdot \bar{G}+\gamma \bar{L}^{H} \cdot \mathcal{D}\left(\bar{W}_{\beta}(\hat{u})\right) \cdot \bar{L}\right)^{-1} \bar{G}^{H}\right] \bar{F}_{\rho},
$$

where $\bar{W}_{\beta}(\hat{u})_{i}=\frac{k}{2}\left(\left(\left|(\bar{L} \cdot \hat{u})_{i}\right|^{2}+\beta\right)^{k / 2-1}\right)$. Starting with an initial guess $\hat{u}^{(0)}$, the nonlinear equation (8) can be solved using the fixed point iteration

$\hat{u}^{(n+1)}=\left[\left(\bar{G}^{H} \cdot \bar{G}+\gamma \bar{L}^{H} \cdot \mathcal{D}\left(\bar{W}_{\beta}\left(\hat{u}^{(n)}\right)\right) \cdot \bar{L}\right)^{-1} \bar{G}^{H}\right] \bar{F}_{\rho}$.

This process is stopped when the difference in the $l_{2}$ norm between successive iterations is small enough, that is, until $\left\|\hat{u}^{(n+1)}-\hat{u}^{(n)}\right\|_{2}^{2} /\left\|\hat{u}^{(n+1)}\right\|_{2}^{2}<\delta$, where $\delta$ is the desired tolerance. In particular, two regularization schemes were studied: (1) total variation regularization (TVR), i.e., $k=1$ and $\bar{L}=\bar{D}$ with $\bar{D}$ the gradient operator, and (2) weighted TVR, i.e., $k=1$ and $\bar{L}=\bar{W} \cdot \bar{D}$ with $\bar{W}$ a matrix that penalizes regions where the object function $\mathcal{O}\left(\vec{r}, \omega_{\max }\right)$ is more uniform. In particular, $\bar{W}$ was set to a two-level function set to 1 at points within $0.75 \lambda$ from the discontinuities of the ideal density profiles, and 10 otherwise.

\section{RESULTS}

\section{A. Simulations}

In order to systematically assess the performance of the MF-DBIM approach, circular cylinders of radii $\lambda, 2 \lambda$, and $4 \lambda$ and excess phase $\Delta \phi_{\max }$ (i.e., the phase accumulated by the incident wave when traveling through the scatterer as opposed to the background) values of $0.9 \pi,-0.45 \pi$, and $0.225 \pi$ were reconstructed through simulations. The density contrast $\Delta \rho$ of the cylinders was varied between $-3 \Delta c$ and $2 \Delta c$, where $\Delta c$ is the speed of sound contrast. $f_{\min }$ was varied between $0.1 f_{\max }$ and $0.9 f_{\max }$. The synthetic data was contaminated with $2 \%$ random Gaussian noise. Five noise realizations per simulation setting were used and the resulting root mean square error (RMSE) mean was calculated. The results are presented in Fig. 1.

The object functions $\mathcal{O}\left(\vec{r}, \omega_{\max }\right)$ corresponding to circular cylinders with radius $2 \lambda, \Delta \phi_{\max }=0.9 \pi$, and $\Delta \rho=-\Delta c$ and $\Delta \rho=\Delta c$ were obtained using DBIM at frequency $f_{\max }$. The $\mathcal{F}_{\rho}(\vec{r})$ profiles were estimated by subtracting the known sound speed dependent term from the reconstructed $\mathcal{O}\left(\vec{r}, \omega_{\max }\right)$ profiles. The ideal profiles for $\mathcal{O}\left(\vec{r}, \omega_{\max }\right)$ and $\mathcal{F}_{\rho}(\vec{r})$ were calculated using (2) with the $\nabla^{2}$ operator implemented using a sinc-based filter. The results are presented in Fig. 2 . 



Fig. 1. Mean RMSEs when reconstructing density profiles of homogeneous circular cylinders with $\Delta \phi_{\max }=0.9 \pi$ (first row), $-0.45 \pi$ (second row), and $0.225 \pi$ (third row), and radii $\lambda$ (left column), $2 \lambda$ (center column) and $4 \lambda$ (right column) using MF-DBIM. The SNR was set to $34 \mathrm{~dB}$
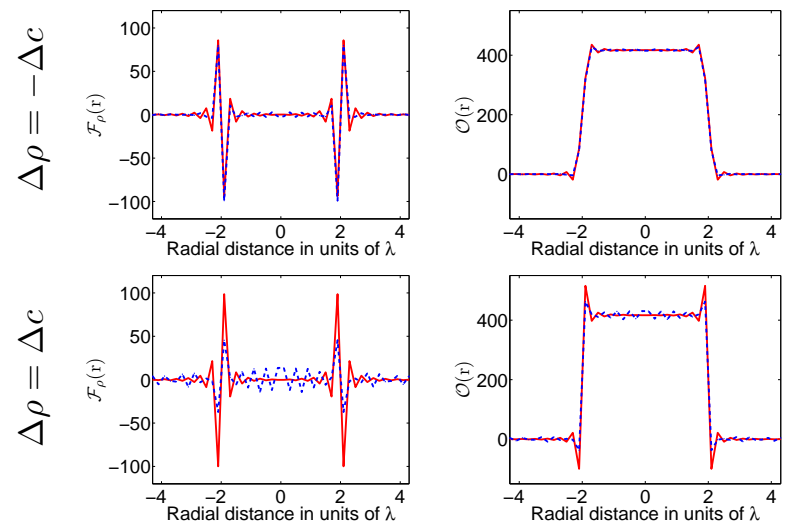

Fig. 2. Expected (solid) and reconstructed (dashed) $\mathcal{F}_{\rho}(\vec{r})$ (left) and $\mathcal{O}\left(\vec{r}, \omega_{\max }\right)$ (right) profiles from circular cylinders of radius $2 \lambda, \Delta \phi_{\max }=$ $0.9 \pi$, and $\Delta \rho=-\Delta c$ (top) and $\Delta \rho=\Delta c$ (bottom).

\section{B. Experiments}

Initial attempts to reconstruct density profiles experimentally were conducted. The imaging target was chosen to be a rubber balloon filled with saline $(6 \mathrm{~g}$ of salt per $100 \mathrm{~mL}$ of water). The resulting speed of sound of the saline was measured to be $1.55 \mathrm{~mm} / \mu$ s using time of flight measurements with two matched $10 \mathrm{MHz}$ transducers. The balloon thickness and speed of sound were measured to be $0.23 \mathrm{~mm}$ and 1.56 $\mathrm{mm} / \mu \mathrm{s}$ using a $20 \mathrm{MHz}$ transducer in reflection mode. The relative density of the balloon was measured to be 0.91 by comparing the amplitude of the reflection from the balloon to that obtained from a plexiglass block. The relative density of the saline solution was measured to be 1.05 by weighing a $10 \mathrm{~mL}$ syringe filled with saline versus water. The outer diameter of the inflated balloon was measured to be $7.6 \mathrm{~mm}$ using calipers. The experiments were performed in water at approximately $22.1^{\circ} \mathrm{C}$. Two independent, single element, rectangular $5 \mathrm{MHz}$ transducers were used for measuring $p^{s c}(\vec{r})$ : one for transmission and one for reception. Both transducers were focused on elevation $\left(f_{\#}=8\right)$ with a nominal focal depth of $8 \mathrm{~cm}$. The transmitter was kept in a fixed location and the receiver was rotated around the imaging target for a total angular coverage of $240^{\circ}$. MF-DBIM reconstructions were obtained by processing scattered data between 1.5 and 3 MHz. The reconstruction error curves are presented in Fig. 3 using both TVR and weighted TVR. The radial profile of the optimum MF-DBIM reconstruction using weighted TVR is also shown.
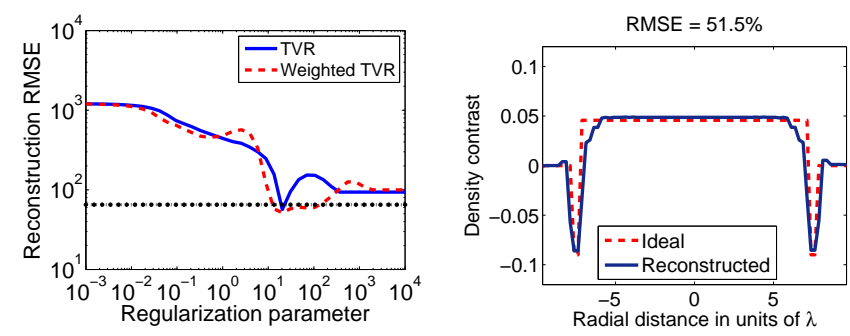

Fig. 3. Left: experimental error curves using MF-DBIM and TVR (solid line) and weighted TVR (dashed line). Right: ideal (dashed line) and optimum reconstructed MF-DBIM and weighted TVR (solid line) density profiles. 


\section{DISCUSSION}

\section{A. Simulations}

A marked difference in the performance of MF-DBIM was observed in Fig. 1 when $\Delta \rho / \Delta c>0$ rather than $\Delta \rho / \Delta c<0$. Whereas in the latter case proper convergence (reconstruction RMSEs $<30 \%$ ) was obtained by using $f_{\min }$ values less than an order of magnitude smaller than $f_{\text {max }}$, in the former case the errors were significantly larger for comparable magnitudes of $\Delta \rho$ changes. In Fig. 2 the reconstructed $\mathcal{F}_{\rho}(\vec{r})$ function for the case $\Delta \rho=-\Delta c$ was very consistent with the expected profile, i.e., sharp discontinuities were observed at the edges of the cylinder. In contrast, and although the expected $\mathcal{F}_{\rho}(\vec{r})$ profile should be almost identical up to a sign change and slight amplitude variation, the $\mathcal{F}_{\rho}(\vec{r})$ estimate when $\Delta \rho=$ $\Delta c$ appeared completely distorted and exhibited ringing that extended beyond the edges of the imaging target.

More insight into the nature of the MF-DBIM reconstruction errors can be obtained by analyzing the $\mathcal{O}(\vec{r})$ plots in Fig. 2. For the $\Delta \rho=-\Delta c$ case, the destructive interaction between the sound speed and density terms resulted in a smooth ideal $\mathcal{O}(\vec{r})$ function and the DBIM properly converged to an approximate solution that accurately captured density effects. In contrast, the ideal $\mathcal{O}(\vec{r})$ function for the $\Delta \rho=\Delta c$ case exhibited sharp variations that DBIM had more difficulties reproducing given its limited spectral support [9]. Increasing $\left|\Delta \phi_{\max }\right|$ made the inverse scattering problem more nonlinear and therefore exacerbated the convergence to improper solutions given the lack of sensitivity of DBIM to high spatial frequencies. This can explain the increased instability of the reconstructions for $\Delta \rho / \Delta c>0$ as $\left|\Delta \phi_{\max }\right|$ increased.

Given that all inverse scattering algorithms based on far field measurements are bandwidth limited, the limitations of MF-DBIM are expected to be shared by other inverse scattering algorithms. The T-matrix approach [3] was used to create density images corresponding to cylinders with $\Delta \phi_{\max }=0.9 \pi$ and radius $2 \lambda$. The reconstruction errors are presented in Fig. 4. The results indicated that the T-matrix approach also has difficulties when imaging homogeneous cylinders with $\Delta \rho / \Delta c>0$, and therefore supported the hypothesis that the spatial bandwidth of the imaging target impacts the performance of tomographic density imaging.

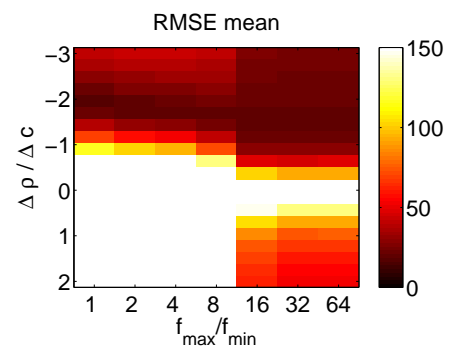

Fig. 4. Mean RMSEs when reconstructing density profiles of homogeneous circular cylinders with $\Delta \phi_{\max }=0.9 \pi$ and radius $2 \lambda$ using the T-matrix approach. The SNR was set to $34 \mathrm{~dB}$.

\section{B. Experiments}

Although the signal-to-noise ratio in the measurements was estimated to be better than $40 \mathrm{~dB}$, the MF-DBIM reconstruction was largely unsuccessful (reconstruction error around $1000 \%$ ). This result suggests that experimental calibration errors may play an important role on the success of density imaging algorithms. Using the same experimental data, both explored regularization schemes resulted in reconstruction errors less than $60 \%$. Although density tomograms with $56 \%$ reconstruction errors were obtained with MF-DBIM and TVR, the range of regularization parameters that resulted in comparable errors was very narrow. Therefore, even a slight error in selecting an appropriate $\gamma_{R}$ value could result in severe reconstruction degradation. In contrast, reconstruction errors between 55\% and 60\% were obtained with MF-DBIM and weighted TVR for regularization parameter values spanning more than an order of magnitude. Therefore, weighted TVR appears to be a more robust mechanism for stabilizing MF-DBIM. Further work is required in order to determine appropriate, general methods to determine $\gamma_{R}$ and $\bar{W}$.

\section{CONCLUSions}

Although MF-DBIM was capable of obtaining density images with reduced noise sensitivity and total bandwidth when compared to previously available methods, its performance degraded when scatterers with large spatial frequency variations in their object function profiles were imaged. However, preliminary experimental results presented here suggest auxiliary techniques such as weighted TVR may help extending the convergence of tomographic density imaging algorithms.

\section{REFERENCES}

[1] D. Borup, S. Johnson, W. Kim, and M. Berggren, "Nonperturbative diffraction tomography via Gauss-Newton iteration applied to the scattering integral equation," Ultrasonic Imaging, vol. 14, no. 1, pp. 69-85, January 1992.

[2] M. J. Berggren, S. A. Johnson, B. L. Carruth, W. W. Kim, F. Stenger, and P. K. Kuhn, "Ultrasound inverse scattering solutions from transmission and/or reflection data," in Proceedings of the SPIE, vol. 671, 1986, pp. $114-121$.

[3] J. Lin and W. Chew, "Ultrasonic imaging by local shape function method with CGFFT," IEEE Transactions on Ultrasonics, Ferroelectrics, and Frequency Control, vol. 43, no. 5, pp. 956-969, September 1996.

[4] R. Lavarello and M. Oelze, "Density imaging using inverse scattering," Journal of the Acoustical Society of America, vol. 125, no. 2, pp. 793802, February 2009.

[5] R. J. Lavarello and M. L. Oelze, "An improved method for tomographic density imaging using a multiple frequency inverse scattering approach," in IEEE Ultrasonics Symposium, 2009, pp. 1467-1470.

[6] W. C. Chew and Y. M. Wang, "Reconstruction of two-dimensional permittivity distribution using the distorted Born iterative method," IEEE Transactions on Medical Imaging, vol. 9, no. 2, pp. 218-225, June 1990.

[7] O. Haddadin and E. Ebbini, "Imaging strongly scattering media using a multiple frequency distorted Born iterative method," IEEE Transactions on Ultrasonics, Ferroelectrics, and Frequency Control, vol. 45, no. 6, pp. 1485-1496, November 1998.

[8] W. C. Karl and M. Cetin, "Feature-enhanced synthetic aperture radar image formation based on nonquadratic regularization," IEEE Transactions on Image Processing, vol. 10, no. 4, pp. 623-631, April 2001.

[9] T. J. Cui, W. C. Chew, X. X. Yin, and W. Hong, "Study of resolution and super resolution in electromagnetic imaging for half-space problems," IEEE Transactions on Antennas and Propagation, vol. 52, no. 6, pp. 1398-1411, June 2004. 